\title{
AN APPROACH TO MODELLING OF PERSONALIZED BONE GRAFTS BASED ON ADVANCED TECHNOLOGIES
}

\author{
Budak, I. ; Mirkovic, S. .*; Sokac, M. ; Santosi, Z. ; Puskar, T. ${ }^{* *} \&$ Vukelic, D. \\ *University of Novi Sad, Faculty of Technical Sciences, Trg Dositeja Obradovica 6, 21000 Novi Sad, \\ Serbia \\ ${ }^{* *}$ University of Novi Sad, Medical Faculty, Hajduk Veljkova 12, 21000 Novi Sad, Serbia \\ E-Mail: budaki@uns.ac.rs, sinisa.mirkovic@uns.ac.rs, marios@uns.ac.rs, zeljkos@uns.ac.rs, \\ tpuskar@uns.ac.rs, vukelic@uns.ac.rs
}

\begin{abstract}
Personalized bone grafts are one of the best examples of the latest achievements in the biomedical engineering. In the area of maxillofacial bone tissue reconstruction or jaw bone augmentation, their application has for some time been on the rise, and its ever increasing significance is driven by the growing technical support. One of the key segments is the bone graft modelling customized to suit patient's specific needs, since it greatly determines not only the future anatomic functionality but also the acceptance probability of the graft by the bone tissue. With the graft geometry importance in mind, presented in this paper is an approach to personalized bone graft modelling. The approach is based on application of modern computer-aided systems and methods, and enables efficient geometric design while minimizing the risk of errors during modelling and placement stages. Verification is based on a case study of a personalized bone graft designed for a patient requiring mandible augmentation.

(Received in January 2016, accepted in June 2016. This paper was with the authors 2 months for 2 revisions.)
\end{abstract}

Key Words: Modelling, Simulation Analysis, Personalized Bone Graft

\section{INTRODUCTION}

Transformation of physical objects into virtual 3D models has recently become extremely important in diverse areas of science and engineering. Computer-aided design (CAD) of virtual models is no longer the exclusive privilege of engineering design, but has been accepted by various domains of medicine and dentistry [1,2]. The reasons for such trend are to be found in the present state-of-the-art in this technology, as well as the ever growing need for fast and high-precision manufacture of parts of human anatomy. Thus, CAD is today being intensively used in the biomedical industry - from the software for generation of virtual anatomical models, to the design of custom-made medical implants, to tissue and bone engineering [3,4]. Bearing in mind the complexity of human anatomy, this has been made possible primarily due to developments in the field of Reverse Engineering (RE) and systems for 3D-digitalization [5-8].

The development of Cone-Beam computerized tomography (CBCT) represents one of the revolutionary innovations in the field of medical and dental 3D-digitalization. Utilization of CBCT has brought maxillofacial and orthodontic diagnostics to a higher level, while the software development brought about revolution in the virtual planning of surgical operations/treatments. In a few years, during which it has been employed, the CBCT proved to be a reliable system for 3D-digitalization of human maxillofacial and dental regions $[9,10]$.

Development and application of additive manufacturing technologies enabled fabrication of parts directly from virtual 3D models, adding to the importance of RE in this domain, simplifying surgical procedures and making them more reliable. Owing to the development of modern technologies for manufacture of bio-compatible materials (CAM systems [11] and advanced additive manufacturing technologies $[12,13])$, the boundaries of RE application have been shifted towards medicine and dentistry. 
Personalized bone grafts are one of the best examples of the current state-of-the-art in this domain. Implementation of such bone grafts in the reconstruction of damaged bone tissue in maxillofacial area or augmentation of jaw bone segments to treat bone resorption or other dental defects has been under development for some time and is gaining importance with the growing technical support. Beside the characteristics of bio-compatible materials, one of the key segments which affect the outcome of therapy is the bone graft design tailored to suit individual requirements. In fact, the importance of design is two-fold - on the one side, bone graft must possess required geometric properties to provide anatomic functionality, while on the other side, the quality of mating with the host bone should diminish the probability of graft rejection by the bone tissue. The manufacture of bone grafts is currently based on cutting technologies and CAM applications. Application of additive or layered manufacturing technologies is still in the research phase $[12,14]$.

The available literature still lacks sufficient information on personalized graft design, dealing mostly with the medical aspects of the problem. The design and manufacturing methods are still addressed briefly, which is understandable considering their recency. Jacotti et al. [15] reported on a clinical case of posterior atrophic mandible rehabilitation with onlay allograft derived from the cancellous bone of femoral heads, manufactured by the application of CAD/CAM technologies in order to assure a perfect fitting of the block above the alveolar crest. Mirkovic et al. reported [16] on augmentation case in right lateral region of the lower jaw by the application of custom-made bone graft, manufactured using CAD/CAM technologies. Another report close to this field is of Mangano et al. [17] in which they presented results of a one-year study of maxillary ridge augmentation with custom-made scaffolds on 10 patients derived from CAD/CAM technologies. Furthermore, Mangano et al. [18] reported on a case of maxillary sinus augmentation using CAD/CAM technologies in order to increase bone height for insertion of dental implants. They developed a technique in order to generate custom-made block grafts for sinus augmentation using CAD/CAM technologies, and a customized cutting guide to precisely position the lateral wall and facilitate membrane elevation, using CBCT. Schlee and Rothamel [19] also demonstrated an application of $\mathrm{CAD} / \mathrm{CAM}$ technologies in designing and manufacturing customized allogenous bone blocks (CABB) out of a single block of bone allograft for horizontal and vertical post-foraminal mandibular defects. The aim of this study was to make possible to evaluate handling and healing patterns of customized allogenic bone blocks for vertical and horizontal alveolar defect augmentation.

Considering the importance of their geometric form, proposed in this paper is an approach to geometric modelling of bone grafts. Based on the application of advanced computer-aided systems and methods, this approach allows efficient generation of geometric form while minimizing risk of error during design and placement stages. Special emphasis is placed on analysis of geometric features of the final 3D graft model and verification of the quality of mating between the graft and the placement location in the mandible. This stage is extremely important for the firm fixation of the designed bone graft to the mandible, which facilitates acceptance of the graft and consequently the success of intervention.

For the purpose of illustration and verification, the proposed approach has been applied to design a bone graft for mandible augmentation in a patient with a pronounced physiological resorption of alveolar ridge.

\section{METHODOLOGY OF THE PROPOSED APPROACH}

\subsection{Modelling procedure}

Surgical implanting of personalized bone graft is a complex task, and recent developments in this field have opened new possibilities for advancement in maxillofacial and oral surgery. 
The success of such surgical procedure depends on several important factors. Among them, most important are: biocompatibility of graft material, its compatibility with the membranes used, and dimensional features (size and thickness) of the graft. The procedure of 3D modelling of a personalized bone graft is complex and sophisticated, since it most often deals with complex surfaces, where every graft has its unique form to suit patient's personal anatomical requirements [1].

Bearing in mind the impact of personalized graft design on the final success of surgical procedure, design methodology has been developed, as shown in Fig. 1. The algorithm represents procedure flow for a personalized 3D bone-graft model design, and defines all the necessary steps which guarantee successful modelling, minimizing the risk of errors in the technical preparation area.

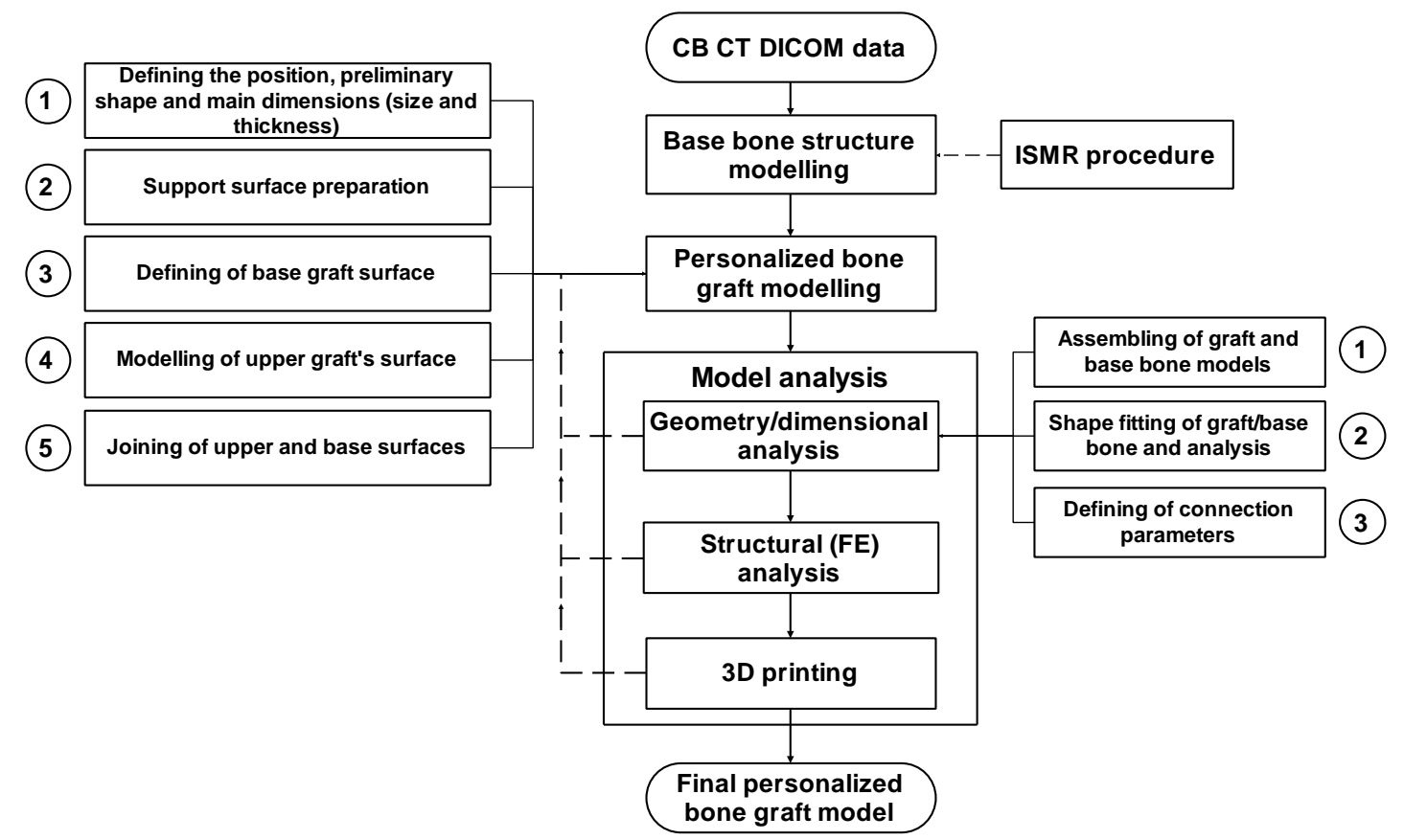

Figure 1: Workflow of the proposed approach.

First step within the modelling task is generation of a 3D base bone model, which will host the future graft. Necessary prerequisite for this step are CBCT images which are imported in DICOM format into the software for analysis and processing of medical imaging. Since the generated 3D model of the base bone structure represents the platform for all other steps of personalized graft modelling, it is crucial to ensure its high geometrical and dimensional accuracy. CBCT images are essential for the accuracy, but they, in turn, depend on several factors. Some of the factors - such as the operating condition of CBCT system, its calibration, and operator skills - can be directly influenced, while the anatomical features of patient's bones remain out of control. This is why, in the process of 3D model generation, the segmentation of DICOM images can be problematic. Most common problems are seen in the segmentation of anatomical areas with pronounced differences in material density (e.g. teeth and jaw bones). In these cases, the use of same set of segmentation parameters (upper and lower threshold values based on Otsu's method) fails to yield satisfactory result regarding geometrical representation of the entire bone structure. The reason lies in the fact that the attempt to set parameters for different intensity pixels, results in pronounced noise levels within particular regions, due to the difference in their densities. This requires a compromise when it comes to pixel intensity threshold, i.e., an optimal value for the pixel intensity range needs to be found in order to generate the 3D model, which, in turn, increases the time required for post-processing. In order to overcome this problem, a procedure has been 
developed for independent segmentation of multiple regions (ISMR). Within this procedure, regions of various densities (e.g. teeth and jaw bones) are segmented independently - each with the set of specifically adjusted parameters - while the complete 3D model is generated by a Boolean union of the regions' borders. This significantly reduces the time required for the post-processing of CT images.

Once the 3D model of the base bone structure is created, the initial parameters required for the 3D modelling of the graft itself are defined. These parameters include: defining the location/region on the base bone structure, preliminary assessment of the required shape of the graft, and its overall dimensions. The parameters are defined interactively, within the multi-disciplinary medical/engineering or dental/engineering team. This ensures the presence of necessary aesthetic and medical characteristics, as well as the technical functionality of the future graft.

Given the fact that the 3D model of the base bone is used for subsequent graft modelling, it is necessary to adequately prepare the mating surface for the future graft on this model. The preparation predominantly involves smoothing out of the potential undulations which are the result of 3D modelling methodology, and the closing of small voids which are mostly the result of bone structure porosity.

Graft modelling begins with a sketch of the mating area on the base bone. Thanks to interactive sketching tools available in specialized CAD software, this step can be efficiently realized through co-operation between medical and technical teams. The selected area on the base bone, also serves as the lower base surface of the graft model. In this way, high mating accuracy between the graft and base bone is ensured, which significantly contributes to efficiency of surgical procedure and lowers the risk of graft rejection.

After the base surface of the graft is formed, the modelling of upper graft surface can be assumed. Since both graft surfaces are free-form, they are almost impossible to model using conventional CAD tools which are used for standard geometric forms. This requires the use of specialized software which allows 3D modelling of free-form surfaces. The modelling of upper graft surface requires careful consideration of the location, shape, thickness, and size of the graft (Fig. 2). As in the case of location, the size and shape (including thickness) of the future graft also have to be defined by a medical doctor/dentist. When designing custom-made bone grafts for maxilla and mandible, besides the anatomic characteristics, the number and the position of the future implants and the optimal therapy plan of the dental suprastructure that will be made as the final restoration, are very important factors for determination of the bone graft dimensions. During processing and modification of graft surfaces, attention should be paid to their edges. Namely, the edges of these surfaces should be well prepared, since they highly impact the adequate connection between the upper and lower graft surfaces, which ultimately defines the shape of the graft 3D model. Shown in Fig. $2 \mathrm{~b}$ and $2 \mathrm{c}$ is the surface before and after processing of edges.

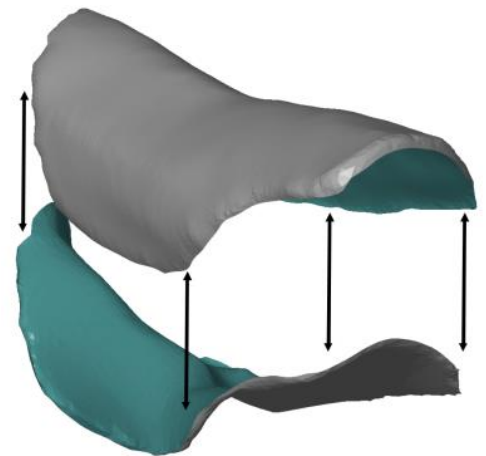

a) modelling and graft's edges

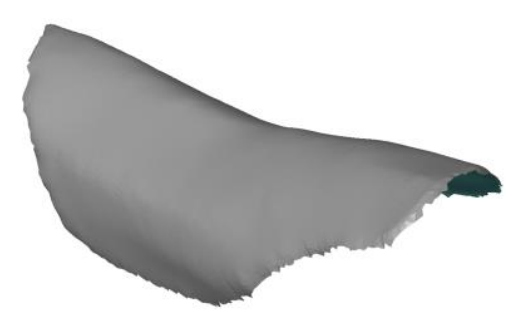

b) before the modification

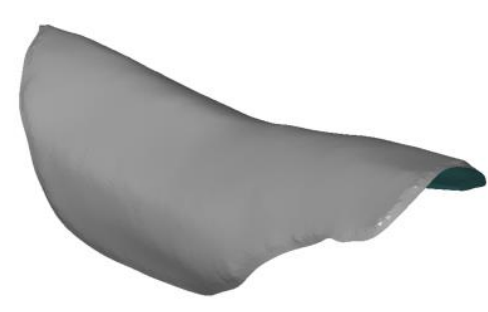

c) after the modification

Figure 2: Sample surface showing. 
Once the upper graft surface modelling has been finished, the upper and base surfaces are being connected. The connection of the two surfaces begins using smaller surfaces, the so called bridges, while the space between them is filled up to form a closed 3D volume. The process of generating satisfactory graft shape is often iterative. The finished 3D graft model is exported into some of the accepted file formats, most often the STL format. This facilitates manipulation with the 3D model and its exchange between CAD/CAE/CAM/RP systems.

\subsection{Model analysis}

Once the 3D model is finished, it is necessary to conduct a detailed analysis of its geometrical and structural characteristics relative to the base bone and other elements which will be used for its fixation. Owing to a growing number of available software solutions (e.g. OnDemand3D, Cybermed Inc.) which enable simulated stitching of complex medical 3D models, analyses of geometric features are now more efficient and reliable. Geometric analysis is conducted through virtual planning of surgical procedure which starts with the graft insertion at the required place on the base bone. Ensuing is the checkup of geometry of this assembly, including the newly formed shape, and the inspection of the quality of mating between the graft and base bone. The geometric analysis finishes with the definition of parameters, most often related to screw fixation which secures the graft to the base bone (the number of screws, their positions, directions, lengths, and diameters).

One of the aspects which require extra attention are the co-ordinate systems of the two 3D models. Considering the fact that they are usually generated in diverse software systems, the co-ordinate systems of 3D models are almost always mismatched, making the assembly generation difficult. Thus, for example, the co-ordinate system of the 3D models generated in 3D DOCTOR, is located in the upper left corner of the CT image, while in the case of 3D models generated in the OnDemand software, co-ordinate system lies at the centre of the middle CT image.

For the sake of simplicity and accuracy of co-ordinate system movement, formulae (1) and (2) have been developed:

$$
\begin{gathered}
T x y=(T d \cdot T r) / 2 \\
T z=(T s \cdot T r) / 2
\end{gathered}
$$

where: $T x y$ - translation along $X$ and $Y$ axis, $T d$ - dimensions of CT slices $(\mathrm{X} \cdot \mathrm{Y}), T r-$ resolution of CT slices, $T z$ - translation along $Z$ axis, $T s$ - total number of CT slices.

Upon assembly, following parameters are checked up: maximum/overall dimensions (length, width, height), presence of negative angles (undermining) on the graft, graft mating, minimum wall thickness of graft, and presence of sharp edges.

For the majority of parameters, the checkup is simplified by the option which allows generation of cross-sections at the positions of interest. In addition, there exist tools for virtual measurements of assembly geometric features - a standard offer of all specialized software solutions in this domain.

Analysis of the discussed parameters leads to appropriate corrections prior to graft fabrication, which diminishes potential risks during surgical procedure to a minimum. In a particular graft cross-section, based on partition planes, one can inspect the mating between the graft and the mandible. Considering the minimum thickness of graft, it should be noted that its thickness should be no less than $3 \mathrm{~mm}$, in order to preserve its mechanical properties which are directly proportional to porosity of graft material. Presence of locations with weaker mechanical characteristics can endanger graft placement due to a possible graft fracture during placement and fixation to the base bone. This not only impedes the surgical procedure, but also increases risks for the patient. Recommendations for the minimum graft 
wall thickness are mostly given by material manufacturers, based on previous testing of mechanical properties.

Special attention should be paid to the presence of negative angles on the graft. This can not only be a problem during graft fabrication, but is also likely to cause difficulties to the surgeon, or even prevent him/her in placing the graft. Experience indicates that negative angles are much more likely to appear in grafts modelled for base bones with higher porosity, such as the maxilla. Craters within a porous bone cause the presence of bumps on the graft base, which is likely to result in negative angles in a graft 3D model.

The analysis of sharp edges on a graft 3D model is required for two reasons. Firstly, the bio-compatible material which makes up the graft is predominantly porous which mostly prevents creation of sharp edges. However, more importantly, sharp edges on the upper graft surface can damage membranes which cover the graft after fixation, which, in turn, can impede the healing of soft tissue and graft admission.

The design of graft 3D model should take into consideration the performance characteristics of the machine which is used for its fabrication. Overall dimensions of the graft 3D model depend on the dimensions and shapes of the available starting block of material (Fig. 3). With this in mind, it should be noted that, presently, there is a lack of standardization in this area, and dimensions and shapes differ according to manufacturer.

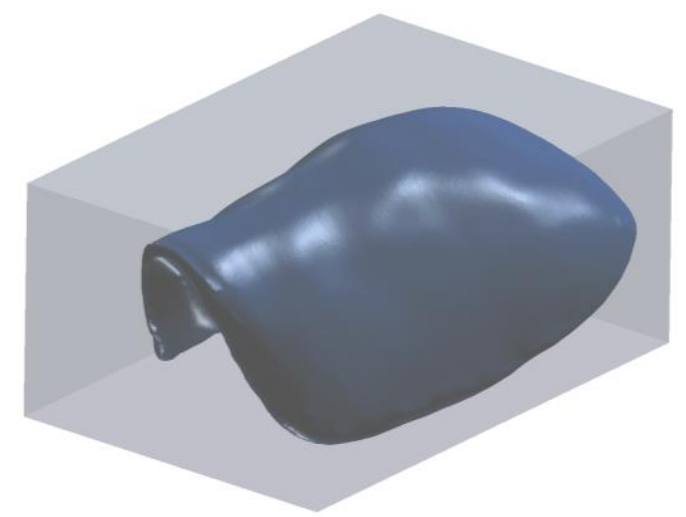

Figure 3: Checking of 3D bone graft model manufacturability based on workpiece dimensions.

If any of the conditions fails to be satisfied, it is necessary to modify the graft 3D model, and then repeat the analysis. Once the graft has satisfied the criteria of virtual analysis, there follows verification on physical models fabricated by some additive manufacturing (AM) technology. This is the last step in the process of quality control before the graft $3 \mathrm{D}$ model is cleared for final manufacture. In this way, it is possible to observe and remove additional potential problems which had escaped the detection on the virtual 3D model [20,21].

$3 \mathrm{D}$ printed model of the graft and base bone lets surgeons gain a better grasp of the physical shape and form, allowing them to adjust the graft to the requirements of the specific situation.

Upon completion of all required modifications and corrections, the graft 3D model is sent to manufacture. To match the geometric complexity of graft 3D model, a 5-axes CNC machining centre is used.

The graft is machined from a single block of bio-compatible material [22], and subsequently sterilized. Beside the most often used box-shaped block, some manufacturers also offer starting shapes in the form of plate, cylinder, and even cone and pyramid.

It should be noted that in recent years a lot of effort has been put into development of AM technologies for 3D printing of bone tissue [20, 21]. The reasons for this trend should be explained by the ability of AM to print porous structures in the designed shape, with controlled chemical composition [12]. It is reasonable to expect that the AM approach shall 
overcome limitations of today's technology for manufacture of complex geometric shapes, rendering the whole process more effective, while increasing the quality of grafts.

\section{RESULTS AND DISCUSSION}

\subsection{Modelling of personalized bone graft}

The developed approach has been practically applied. This is presented in the case study of personalized graft modelling for augmentation of mandible, with the aim to create conditions for implant placement. The first challenge was to model the base bone structure based on CBCT images, using the previously discussed ISMR procedure. Shown in Table I are parameters used for independent segmentation of teeth and mandible. The table lists minimal and maximal threshold values of pixel density used, as well as the number of images that contain contours of teeth and mandible, to which these parameters pertain. Parallel displays of the 3D mandible models generated in 3D DOCTOR software - without ISMR (Fig. 4, left), and with ISMR procedure applied (Fig. 4, right), clearly illustrate its benefit.

Table I: Parameters used for segmentation

\begin{tabular}{|l|c|c|c|}
\cline { 3 - 4 } \multicolumn{1}{c|}{} & \multirow{2}{*}{ Number of images } & \multicolumn{2}{c|}{ Threshold value } \\
\cline { 3 - 4 } \multicolumn{1}{c|}{} & & Minimum & Maximum \\
\hline Teeth & $108-140$ & 2177 & 4096 \\
\hline Mandible & $0-155$ & 1470 & 4096 \\
\hline
\end{tabular}
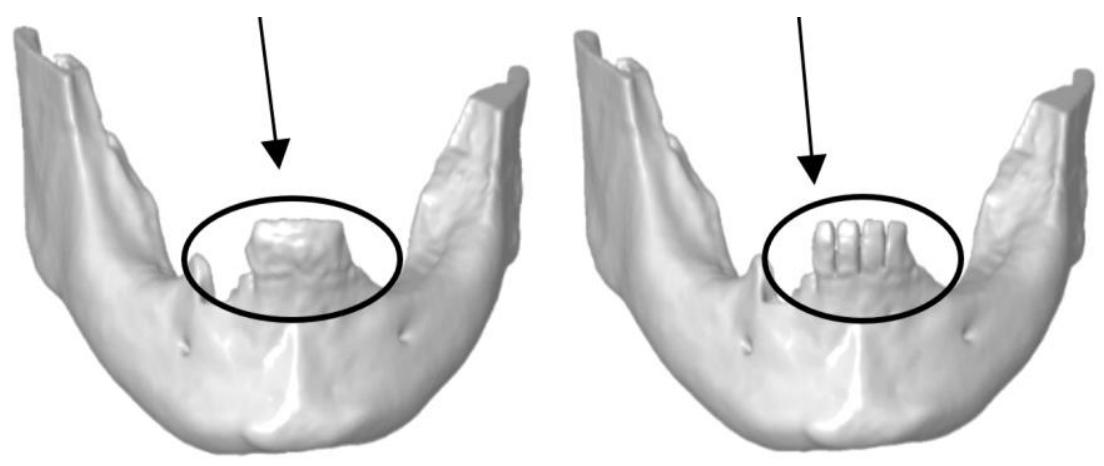

Figure 4: 3D models of mandible with teeth created without (left) and with (right) application of procedure for independent segmentation of multiple regions (ISMR).

Preparation of graft insertion place, i.e., the mating surface with the future graft on the 3D mandible model, is shown in Fig. 5. Graft modelling began by sketching the graft supporting region on the mandible (Fig. 6 a), based on which base surface of the future graft was automatically generated (Fig. 6 b).

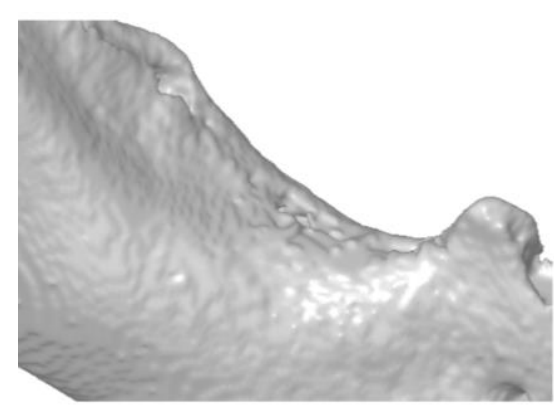

a) before modification

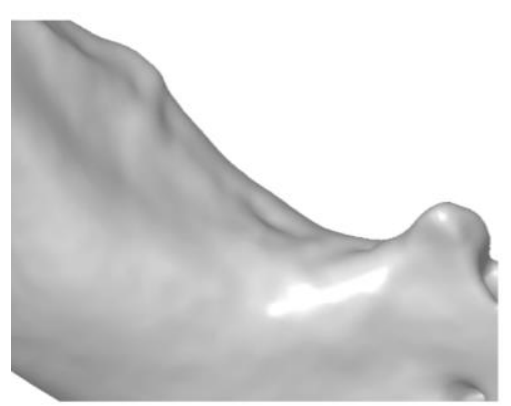

b) after modification

Figure 5: Place of graft insertion. 


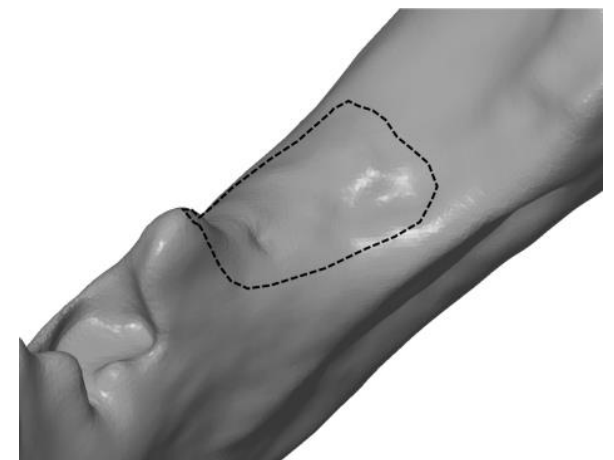

a) sketched supporting region for graft fitting

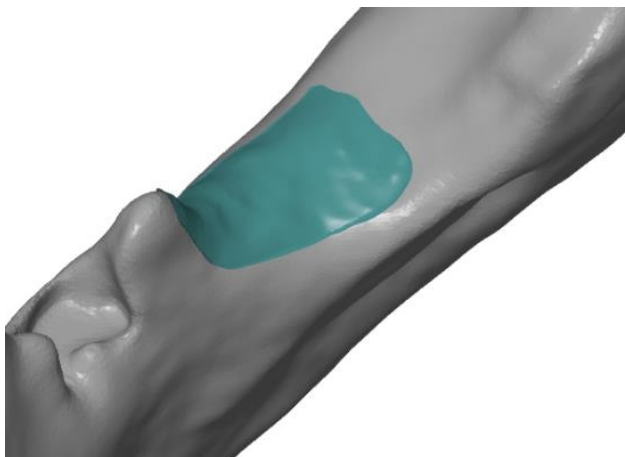

b) modelling of graft's base surface

Figure 6: Showing of graft modelling.

Then the upper graft surface was modelled, and the two surfaces were joined together using the so called "bridges" (Fig. 7). Once the space between the bridges was filled up, the 3D graft model was complete (Fig. 8).

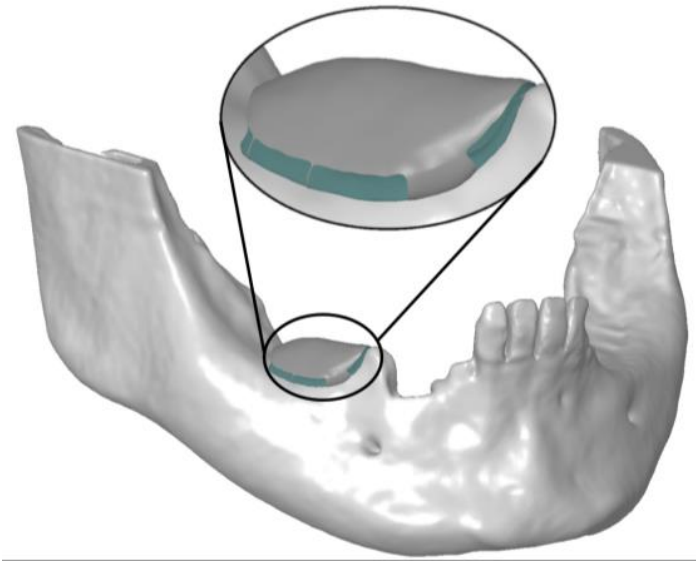

Figure 7: The procedure of joining the base and upper graft surfaces.
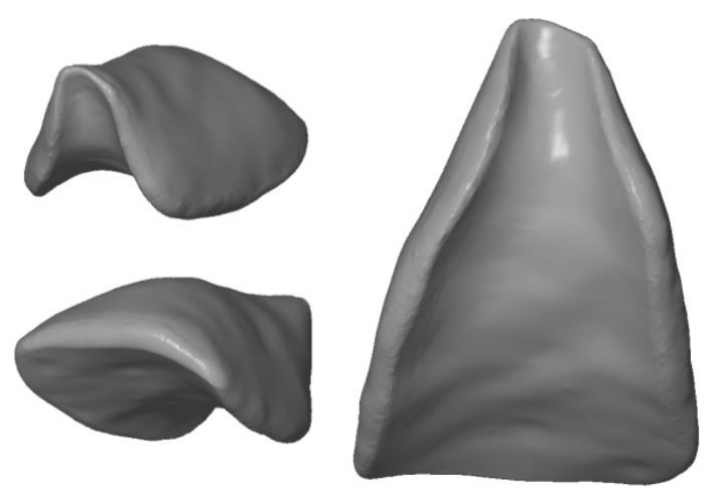

Figure 8: Completed 3D graft model.

\subsection{Model analysis of personalized bone graft}

The first step in the analysis of the completed model is the formation of 3D assembly model of the mandible and graft. Shown in Fig. 9 are the assembly and the planned position and type of implant to be inserted after the successful augmentation.

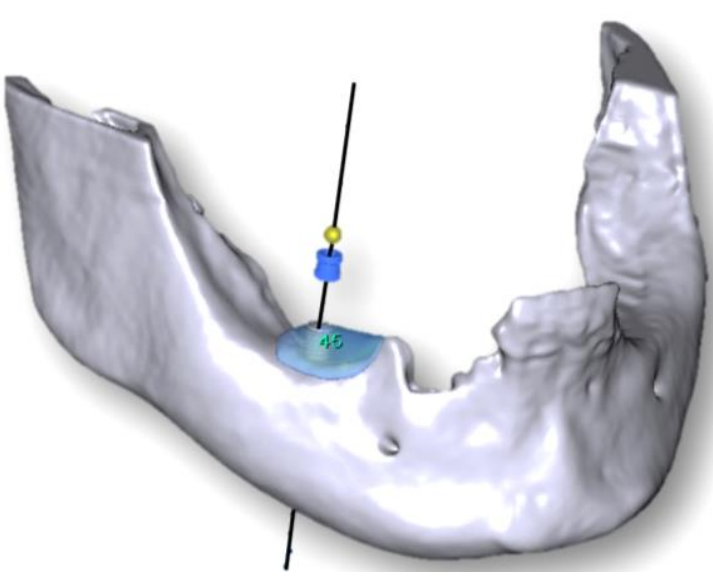

Figure 9: 3D graft model placed on the base bone with the planned position of implant.
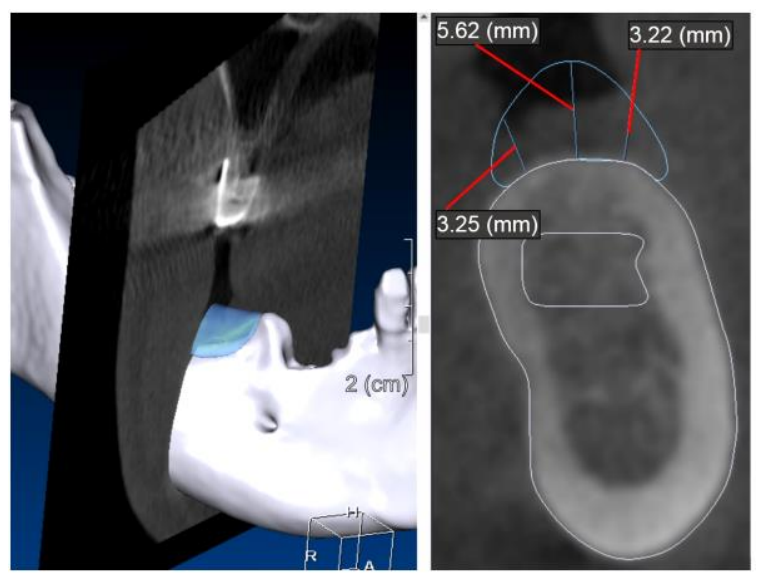

Figure 10: Cross-sectional view of the graft-base bone assembly with virtually measured dimensions. 
The checkup of geometrical parameters was realized using the OnDemand software, based on the assembly cross-sections of interest and the virtual measurement tools (Fig. 10). The cross-section shows that the graft mates very well with the base bone, while the dimensional parameters are within tolerances, without the presence of negative angles and sharp edges.

Following the geometric checkup is the FEM structural analysis of the graft. In the analysis of the modelled mandible graft, the forces generated at the location where the titanium screw fixates the bone graft, were analysed in more detail. Fig. 11 a shows 3D model of the bone graft connected to the mandible with two titanium screws, where "E1" and "E2" represent critical zones of the bone graft around the screws. Fig. $11 \mathrm{~b}$ FE shows the mesh with 58,108 tetrahedral elements and 11,851 nodes. As can be seen from Fig. $11 \mathrm{~b}$, the mesh is more refined for the bone graft, and, compared to the bone, consists of significantly larger number of FEs. The bone graft is connected to the bone by two titanium screws, and the bone itself is fixed by two supports on the sides. This is necessary in order to fulfil all conditions needed to conduct FEM analysis. The mechanical characteristics of Ti-6Al-7Nb screws used for the FEM analysis are as follows: Young modulus, $E=110 \mathrm{GPa}$, the Poisson ratio, $m=0.37$, and density, $q=4530 \mathrm{~kg} / \mathrm{m}^{3}$. For the cortical bone, the mechanical characteristics are: Young modulus, $E=110 \mathrm{GPa}$, the Poisson ratio, $m=0.37$, and density, $q=4530 \mathrm{~kg} / \mathrm{m}^{3}$. For the bone graft, the mechanical characteristics are: Young modulus is $E=3.45 \mathrm{GPa}$, the Poisson ratio, $m=0.31$, and density, $q=1650 \mathrm{~kg} / \mathrm{m}^{3}$. The FE analyses for both screws were performed with three different levels of static preloads of $25 \mathrm{~N}, 37 \mathrm{~N}$ and $50 \mathrm{~N}$.

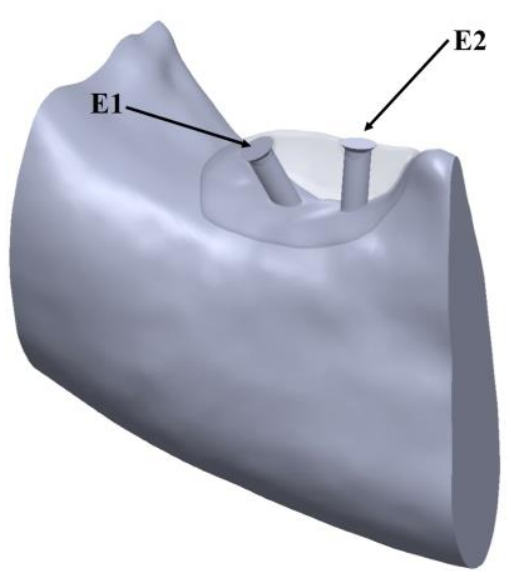

a) bone graft connected to the jaw bone with two titanium screws

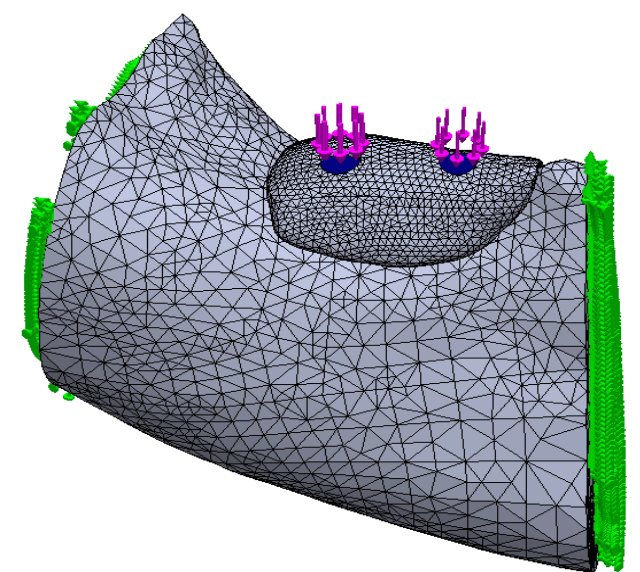

b) FE mesh with 58,108 tetrahedral elements and 11,851 nodes

Figure 11: 3D model.

High stress concentrations were found on the heads of the screws, and the top surface of the bone graft (Fig. 12). The preload is transferred from the screws' countersunk heads onto the top surface of the bone graft. Simulation of various preload directions also leads to conclusion that the stress in zone "E2" is largest for the shown direction, equalling $1.679 \mathrm{e}-011 \mathrm{MPa}$. The stress in "E2" zone is largest when the simulated preload has vertical (Z-axis) direction. The stress zone "E1" shows lower stress values than the stress zone "E2". One of the reasons why the zone "E1" exhibited higher stress is most likely the bone ridge located near that fixation site.

The resulting displacements (Fig. 13) show that most of displacements occur within the stress zone "E2". It is important to consider the distribution of displacements from that stress zone, visible in Fig. 13, which moves up to the bone ridge, indicating a risk of possible breakage of the bone graft.

Upon successful structural analysis, physical models were fabricated using 3D printing technology (Fig. 14). This allowed physical verification of graft model validity by a surgeon. 
$25 \mathrm{~N}$
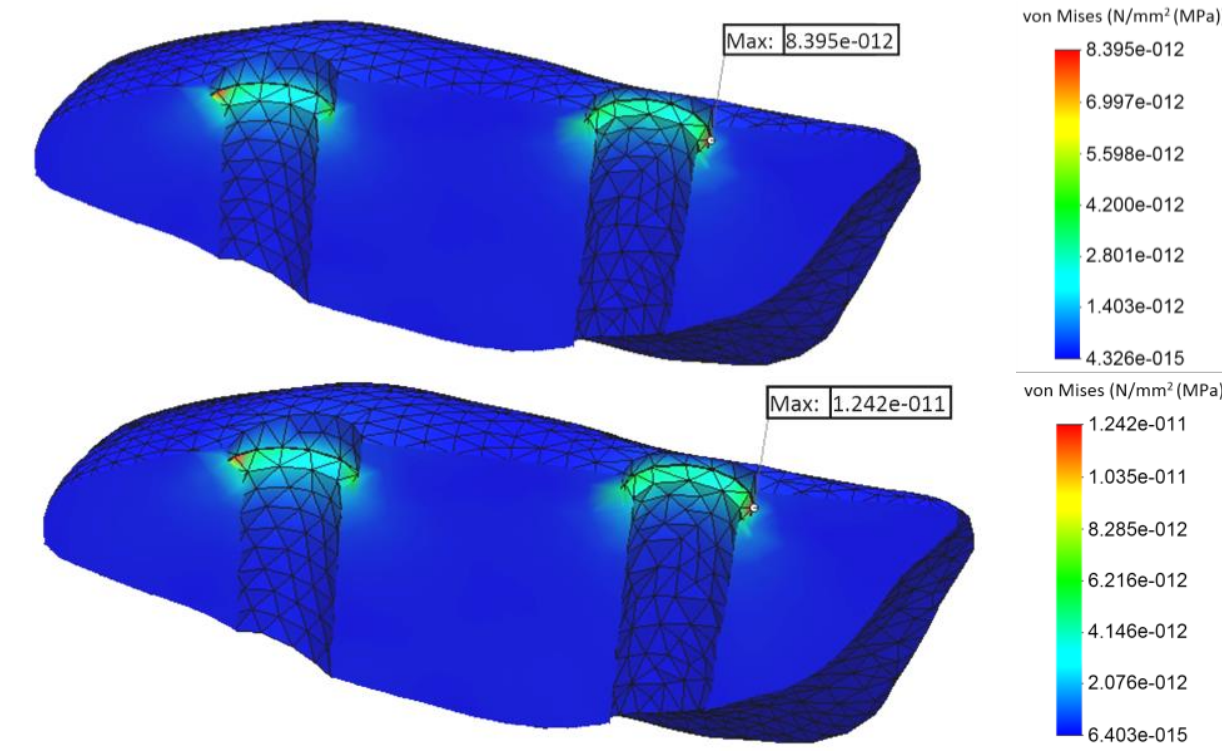

von Mises (N/mm² (MPa))

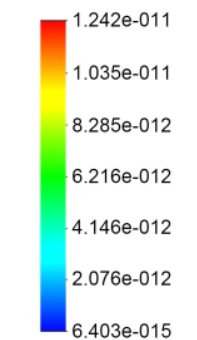

$37 \mathrm{~N}$

von Mises (N/ $\mathrm{mm}^{2}$ (MPa)
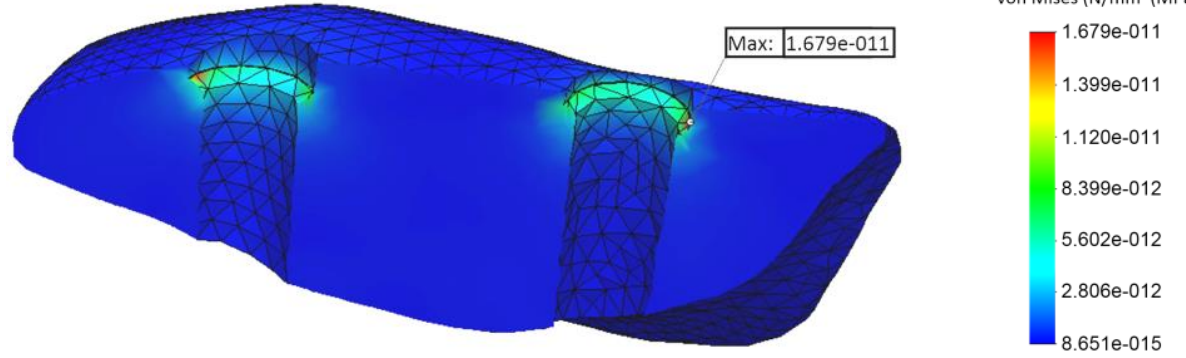

Figure 12: Distribution of Von-Mises stress in the bone graft for different preloads.

$25 \mathrm{~N}$
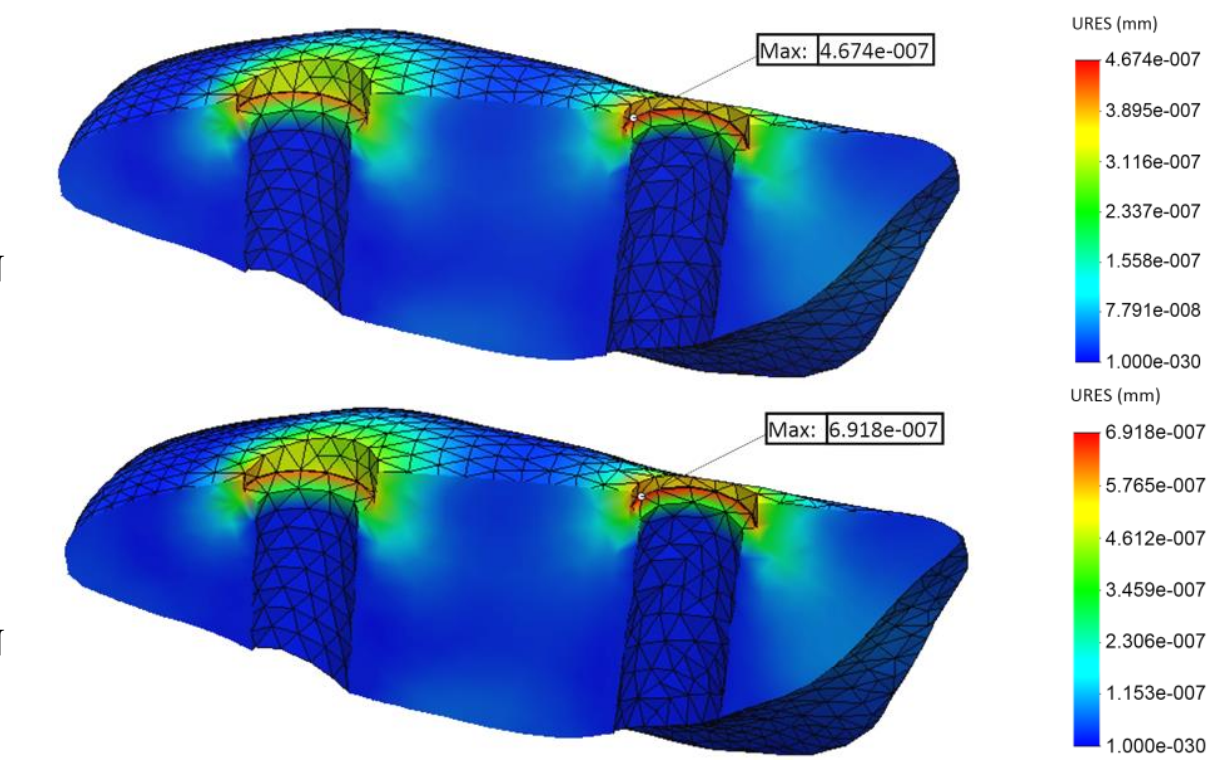

$37 \mathrm{~N}$

URES ( $\mathrm{mm}$ )
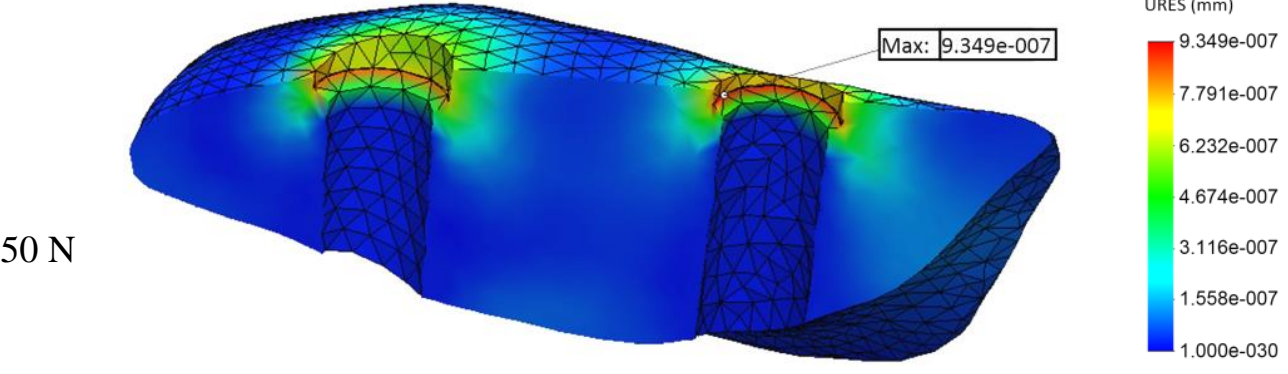

Figure 13: Distribution of resultant displacements in the bone graft for different preloads. 

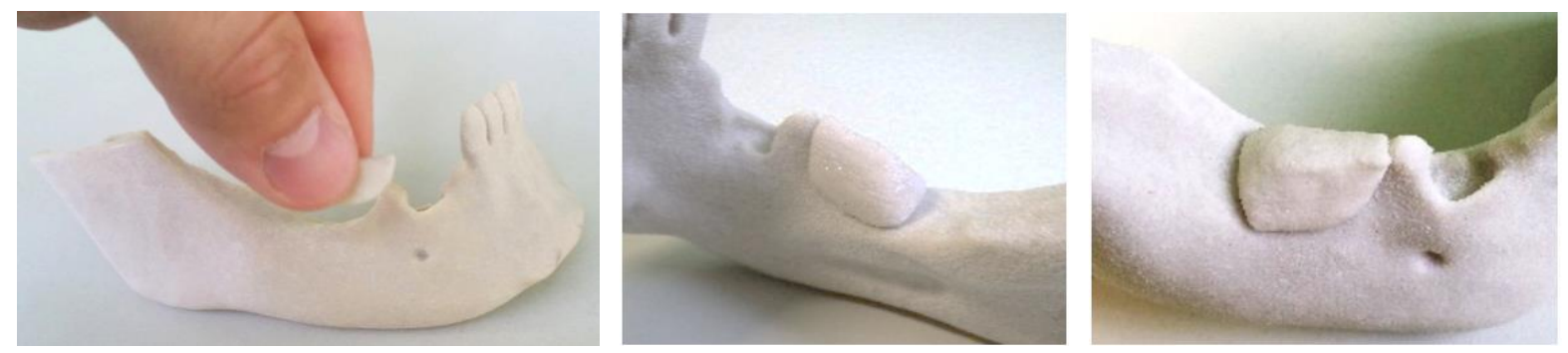

Figure 14: 3D printed models of mandible and bone graft.

\section{CONCLUSION}

Presented in this study is a detailed approach to modelling of personalized bone grafts for use in oral and maxillofacial surgery. The innovation of the proposed approach lies in its integral character, as well as in several specifically designed procedures. The importance of this approach is primarily the applied methodology, and the parameters it uses. The results have confirmed functionality and efficiency of the developed approach, as well as the importance of the parameters identified within particular procedures. Most crucial of those parameters are the position and shape of the graft relative to the required number of implants on the given location, which directly defines the size of the graft. Another important aspect is the graft base surface, which interfaces the base bone, and plays a pivotal role in the success of the surgical procedure and patient recovery. Finally, structural FEM analysis of the modelled graft represents an important segment, which reduces to a minimum the risk of graft failure during surgical procedure.

In all, the proposed approach represents an important step towards enabling closer and more intensive interdisciplinary co-operation between engineering and medical/dental domain. In this context, further investigations shall be focused on the development of knowledge bases, to allow automation of particular procedures within the proposed approach.

\section{ACKNOWLEDGEMENT}

The results presented in this paper are obtained in the framework of the project "Research and development of modelling methods and approaches in manufacturing of dental recoveries with the application of modern technologies and computer aided systems" (TR 35020), financed by the Ministry of Education, Science and Technological Development of the Republic of Serbia.

\section{REFERENCES}

[1] Sun, W.; Starly, B.; Nam, J.; Darling, A. (2005). Bio-CAD modeling and its applications in computer-aided tissue engineering, Computer-Aided Design, Vol. 37, No. 11, 1097-1114, doi:10.1016/j.cad.2005.02.002

[2] Vujica Herzog, N.; Vujica Beharic, R.; Beharic, A.; Buchmeister, B. (2014). Ergonomic analysis of ophthalmic nurse workplace using 3D simulation, International Journal of Simulation Modelling, Vol. 13, No. 4, 409-418, doi:10.2507/IJSIMM13(4)2.265

[3] Foley, B. D.; Thayer, W. P.; Honeybrook, A.; McKenna, S.; Press, S. (2013). Mandibular reconstruction using Computer-Aided Design and Computer-Aided Manufacturing: An analysis of surgical results, Journal of Oral and Maxillofacial Surgery, Vol. 71, No. 2, e111-e119, doi: $10.1016 /$ j.joms.2012.08.022

[4] Parthasarathy, J. (2014). 3D modelling, custom implants and its future perspectives in craniofacial surgery, Annals of Maxillofacial Surgery, Vol. 4, No. 1, 9-18, doi:10.4103/22310746.133065

[5] Budak, I.; Sokovic, M.; Barisic, B. (2011). Accuracy improvement of point data reduction with sampling-based methods by fuzzy logic-based decision-making, Measurement, Vol. 44, No. 6, 1188-1200, doi:10.1016/j.measurement.2011.03.026 
[6] Giordano, M.; Ausiello, P.; Martorelli, M. (2012). Accuracy evaluation of surgical guides in implant dentistry by non-contact reverse engineering techniques, Dental Materials, Vol. 28, No. 9, e178-e185, doi:10.1016/j.dental.2012.06.006

[7] Budak, I.; Trifkovic, B.; Puskar, T.; Vukelic, D.; Vucaj-Cirilovic, V.; Hodolic, J.; Todorovic, A. (2013). Comparative analysis of 3D digitization systems in the field of dental prosthetics, Tehnicki Vjesnik - Technical Gazette, Vol. 20, No. 2, 291-296

[8] Cajal, C.; Santolaria, J.; Samper, D.; Garrido, A. (2015). Simulation of laser triangulation sensors scanning for design and evaluation purposes, International Journal of Simulation Modelling, Vol. 14, No. 2, 250-264, doi:10.2507/IJSIMM14(2)6.296

[9] Agrawal, J. M.; Agrawal, M. S.; Nanjannawar, L. G.; Parushetti, A. D. (2013). CBCT in orthodontics: The wave of future, Journal of Contemporary Dental Practice, Vol. 14, No. 1, 153 157, doi:10.5005/jp-journals-10024-1291

[10] Nervina, J. M. (2012). Cone beam computed tomography use in orthodontics, Australian Dental Journal, Vol. 57, No. Suppl. 1, 95-102, doi:10.1111/j.1834-7819.2011.01662.x

[11] Klancnik, S.; Ficko, M.; Balic, J.; Pahole, I. (2015). Computer vision-based approach to end mill tool monitoring, International Journal of Simulation Modelling, Vol. 14, No. 4, 571-583, doi:10.2507/IJSIMM14(4)1.301

[12] Bose, S.; Vahabzadeh, S.; Bandyopadhyay, A. (2013). Bone tissue engineering using 3D printing, Materials Today, Vol. 16, No. 12, 496-504, doi:10.1016/j.mattod.2013.11.017

[13] Zivic, F.; Grujovic, N.; Manivasagam, G.; Richard, C.; Landoulsi, J.; Petrovic, V. (2014). The potential of magnesium alloys as bioabsorbable / biodegradable implants for biomedical applications, Tribology in Industry, Vol. 36, No. 1, 67-73

[14] Ciocca, L.; De Crescenzio, F.; Fantini, M.; Scotti, R. (2009). CAD/CAM and rapid prototyped scaffold construction for bone regenerative medicine and surgical transfer of virtual planning: A pilot study, Computerized Medical Imaging and Graphics, Vol. 33, No. 1, 58-62, doi:10.1016/j.compmedimag.2008.10.005

[15] Jacotti, M.; Barausse, C.; Felice, P. (2014). Posterior atrophic mandible rehabilitation with onlay allograft created with CAD-CAM procedure: A case report, Implant Dentistry, Vol. 23, No. 1, 22-28, doi:10.1097/id.0000000000000023

[16] Mirkovic, S.; Budak, I.; Puskar, T.; Tadic, A.; Sokac, M.; Santosi, Z.; Djurdjevic-Mirkovic, T. (2015). Application of modern computer-aided technologies in the production of individual bone graft: A case report, Vojnosanitetski pregled, Vol. 72, No. 12, 1126-1131, doi:10.2298/ VSP140915117M

[17] Mangano, F.; Macchi, A.; Shibli, J. A.; Luongo, G.; Iezzi, G.; Piattelli, A.; Caprioglio, A.; Mangano, C. (2014). Maxillary ridge augmentation with custom-made CAD/CAM scaffolds. A 1-year prospective study on 10 patients, Journal of Oral Implantology, Vol. 40, No. 5, 561-569, doi:10.1563/aaid-joi-d-12-00122

[18] Mangano, F.; Zecca, P.; Pozzi-Taubert, S.; Macchi, A.; Ricci, M.; Luongo, G.; Mangano, C. (2013). Maxillary sinus augmentation using computer-aided design/computer-aided manufacturing (CAD/CAM) technology, International Journal of Medical Robotics and Computer Assisted Surgery, Vol. 9, No. 3, 331-338, doi:10.1002/rcs.1460

[19] Schlee, M.; Rothamel, D. (2013). Ridge augmentation using customized allogenic bone blocks: Proof of concept and histological findings, Implant Dentistry, Vol. 22, No. 3, 212-218, doi:10.1097/id.0b013e3182885fa1

[20] Budzik, G.; Burek, J.; Bazan, A.; Turek, P. (2016). Analysis of the accuracy of reconstructed two teeth models manufactured using the 3DP and FDM technologies, Strojniski vestnik - Journal of Mechanical Engineering, Vol. 62, No. 1, 11-20, doi:10.5545/sv-jme.2015.2699

[21] Goffard, R.; Sforza, T.; Clarinval, A.; Dormal, T.; Boilet, L.; Hocquet, S.; Cambier, F. (2013). Additive manufacturing of biocompatible ceramics, Advances in Production Engineering \& Management, Vol. 8, No. 2, 96-106, doi:10.14743/apem2013.2.157

[22] Pantic, M.; Mitrovic, S.; Babic, M.; Jevremovic, D.; Kanjevac, T.; Dzunic, D.; Adamovic, D. (2015). AFM surface roughness and topography analysis of lithium disilicate glass ceramic, Tribology in Industry, Vol. 37, No. 4, 391-399 